\title{
BMJ Open Living with pulmonary hypertension: unique insights from an international ethnographic study
}

\author{
Martha Kingman, ${ }^{1}$ Barbara Hinzmann, ${ }^{2}$ Oliver Sweet, ${ }^{3}$ Jean-Luc Vachiéry ${ }^{4}$
}

To cite: Kingman $\mathrm{M}$, Hinzmann B, Sweet 0, et al. Living with pulmonary hypertension: unique insights from an international ethnographic study. BMJ Open 2014;4:e004735. doi:10.1136/bmjopen-2013004735

- Prepublication history for this paper is available online. To view these files please visit the journal online (http://dx.doi.org/10.1136/ bmjopen-2013-004735).

Received 20 December 2013 Revised 31 March 2014 Accepted 17 April 2014

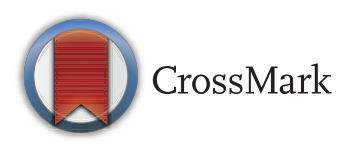

${ }^{1}$ Pulmonary Hypertension Program, UT Southwestern Medical Center, Dallas, Texas, USA

${ }^{2}$ Department of Global Market Research, Market Research Cardiology, Bayer HealthCare Pharmaceuticals, Berlin, Germany

${ }^{3}$ Ethnography Centre of Excellence, Ipsos MORI, London, UK

${ }^{4}$ Département de Cardiologie, Cliniques Universitaires de Bruxelles, Hôpital Erasme, Brussels, Belgium

Correspondence to Dr Martha Kingman, Martha.Kingman@

UTSouthwestern.edu

\section{ABSTRACT}

Objectives: To better understand the patient's perspective of pulmonary hypertension ( $\mathrm{PH})$, including the impact of living with $\mathrm{PH}$, disease management and treatment.

Design: This qualitative ethnographic study collected observational video footage, supplemented by field notes and patient diaries to assess the impact of $\mathrm{PH}$ on the patient's life.

Setting: Patients were observed and filmed in their home for up to $6 \mathrm{~h}$, capturing the environment, interactions and activities of everyday life.

Participants: Patients with pulmonary arterial hypertension (PAH) or chronic thromboembolic $\mathrm{PH}$ who were receiving $\mathrm{PAH}$-specific medication were recruited through healthcare professionals (HCPs) and patient associations in seven countries across four continents. Sampling was purposive and subgroup analysis was not intended.

Results: Overall, 39 patients with PH were enrolled. Many patients had a poor understanding of $\mathrm{PH}$ and found their 'invisible' disease difficult to explain to others. An important finding was the secrecy surrounding PH. Feelings of insecurity and isolation were regularly reported, and many patients admitted to hiding their symptoms. The marked improvement in symptoms after therapy initiation made assessment of disease progression more difficult as patients compared their quality of life ( $Q \mathrm{oL}$ ) against pretreatment levels. Extensive planning and adherence to daily routines were required in patients' everyday life.

Conclusions: Ethnography was used for the first time, in several countries, to evaluate the patient's perception of living with PH. This approach revealed key findings that would not typically be uncovered using other qualitative techniques, including the secrecy surrounding $\mathrm{PH}$, the difficulties in describing the disease and the challenges in assessing disease progression. A more tailored dissemination of information from HCPs and development of a simple and understandable $\mathrm{PH}$ definition may be beneficial in alleviating the secrecy reported by patients. A greater appreciation of how patients perceive their disease and $\mathrm{QoL}$ has the potential to improve $\mathrm{PH}$ management.

\section{INTRODUCTION}

Pulmonary hypertension $(\mathrm{PH})$ is a group of heterogeneous diseases, defined by an

\section{Strengths and limitations of this study}

- This is the first ethnographic study to investigate the experiences of patients living with pulmonary hypertension and uncovers a number of findings that would not typically be revealed by other qualitative approaches.

- The study included an international sample of patients with an array of demographic features in order to provide a wide range of perspectives on living with the disease and to identify trends.

- A larger study including more patients across additional countries is warranted to allow subanalyses to be undertaken according to demographic variables, country and disease type.

increase in mean pulmonary artery pressure $\geq 25 \mathrm{~mm} \mathrm{Hg}$ measured by right heart catheterisation. ${ }^{1}$ Pulmonary arterial hypertension (PAH), a subtype of $\mathrm{PH}$, is a chronic disease, characterised by vasoconstriction and vascular proliferation of the small pulmonary arteries, eventually leading to right heart failure and death. ${ }^{1}$ Despite the availability of several specific therapies, $\mathrm{PAH}$ is severely disabling and carries a poor prognosis. ${ }^{2}$ Chronic thromboembolic PH (CTEPH) is another subtype of $\mathrm{PH}$, which develops from the obstruction of pulmonary artery branches following chronic pulmonary thrombosis, leading to the formation of fibrosis and remodelling of pulmonary blood vessels. ${ }^{1}$ Pulmonary endarterectomy is the only curative therapy for CTEPH; however, approximately $35 \%$ of patients are not eligible for this surgery and such patients have a poor prognosis, similar to those with PAH. ${ }^{3-5}$

The physically and psychologically disabling natures of PAH and CTEPH can severely impact patients' relationships with family and friends, their ability to work and exercise, and their financial security. ${ }^{6}$ Consequently, quality of life (QoL) assessments are often included as secondary endpoints in clinical studies to investigate the effectiveness of $\mathrm{PH}$ medications. A variety of tools have been developed, including generic measurements 
such as SF-36 and EuroQoL, disease-specific tools such as Cambridge Pulmonary Hypertension Outcome Review (CAMPHOR) and Minnesota Living with Heart Failure Questionnaire (MLHFQ), and symptom-specific measures such as the Borg dyspnoea scale. ${ }^{6}{ }^{7}$ However, despite the relatively widespread use of such assessments, they mainly focus on the physical and functional status of patients and do not evaluate specifically how patients perceive their disease.

In the present study, a qualitative technique based on the observation of patient behaviour was used to gain a better understanding of the beliefs and practices associated with living with $\mathrm{PH}$ from the patient's perspective. Ethnography, which has its roots in social anthropology, typically uncovers findings that would not normally be revealed by other qualitative approaches. Ethnographic methodology places the researcher in the participant's environment, directly observing actual behaviour for an extended length of time, capturing the interactions and activities of everyday life. This technique identifies potential disconnects between actual behaviour and reported behaviour of patients. In doing so, it places importance on the mundane elements of life that patients are less likely to report, including behaviours that have become embedded in everyday routines. Commonly employed observational recording methods include field notes, audio recordings and video recordings. Ethnography has been used previously in public health research ${ }^{8}$ but has rarely been applied to the in-depth study of healthcare issues. ${ }^{9}$ We have applied this established methodology to a healthcare setting to focus on three key research objectives: to gain a holistic understanding of the impact that $\mathrm{PH}$ has on patients; to explore disease management and treatment from a patient's perspective; and to fully understand the patient-healthcare professional (HCP) relationship. In order to obtain a wide range of perspectives on living with $\mathrm{PH}$, we aimed to include patients with $\mathrm{PAH}$ or CTEPH of varying severities, from several different countries and with a wide range of demographic features. To the best of our knowledge, this is the first study to apply these methods in PH.

\section{METHODS}

Patient recruitment

This qualitative study included patients aged $\geq 18$ years who had been diagnosed with PAH or CTEPH for $\geq 6$ months and were receiving PAH-specific medication. Patients were given detailed study information by their HCPs and patient associations (organisations that provide a number of services to the $\mathrm{PH}$ community, including support, education, research and advocacy; table 1), and could contact the research agency if they were interested in participating. Such patients were then screened for the necessary inclusion criteria. Sampling was purposive with 'soft quotas' set on the types of patients to take part to ensure, where possible, that a mix of ages, gender, type of

\begin{tabular}{ll} 
Table 1 Patient recruitment & \\
\hline Country & Patients recruited through \\
\hline Brazil & Patient associations \\
France & Physicians \\
Germany & Physicians \\
Italy & Patient associations \\
Republic of Korea & Patient associations \\
UK & Specialist pulmonary \\
USA & hypertension nurses \\
\hline
\end{tabular}

PAH-specific medication and socio-economic grades were included from each of the seven countries (Brazil, France, Germany, Italy, Republic of Korea, UK and USA). Prior to enrolment, eligible patients were given details of the study and written informed consent was obtained, in adherence to local recruitment laws. All patients were telephoned by the researcher before the research started in order to establish a good rapport. If requested, patients had access to the identity of the sponsor at the end of the study.

\section{Data collection}

Ethnography focuses on examining the context and environment in which everyday decisions are made. The study used discussions and observations to assess real-life patterns of behaviour. UK-based specialist ethnographers employed by Ipsos MORI (London, UK) carried out the research. All researchers were native speakers of the local language in the country in which the research took place. Researchers observed and filmed the patients in their homes for up to $6 \mathrm{~h}$ with a small hand-held camera, capturing the environment, interactions and activities of everyday life and looking for the behaviour and context in which patients made decisions. The researchers had a loose discussion guide (observation matrix) but were guided by what the patient had planned to do on the day of the visit. The emphasis was on watching and listening, rather than questioning. In addition to recording the patient's responses on video, researchers prepared detailed field notes.

Diaries were provided to the patients at the point of recruitment and patients were asked to complete them before the home visit to gain a better understanding of the patient's perspective of their illness. The patients were not provided with a guide for completing the diaries. Projective and creative techniques were applied to ensure that patients were comfortable describing themselves, and included four written tasks titled: my perfect day, how my treatment makes me feel, letter to my doctor and if my illness was a person (box 1). These techniques offered a structure by which patients could access thoughts, emotions or needs that were difficult to verbalise or express publicly and were not easily accessible to the patients themselves and/or to the researcher. The responses were analysed alongside ethnography 
Box 1 Example of a patient's response to the written task in their patient diary titled 'if my illness was a person'.

If my illness was a person...

- "Whenever my lung pressure is high, it feels dizzy, feels like all the blood veins are expanded and the upper body is on fire. Breathing becomes hard and lung seems to not function at all".

- "Rotten lung without function".

- "At stage 4, it feels like carrying a heart and a lung that are outside of the body. How dangerous is it to carry the important organs? They are like bombs on fire, you don't know when they will explode".

- "Sigh a lot naturally because it is stuffy".

"Heart that is about to burst".

- "Cold only around the navel".

- "With no exercise, lower body loses strength. It's like ET's body".

- "Not eating well, lack of sunlight and always lying down due to PH caused osteoporosis".

- "Feels like always carrying sandbags, feels heavy".

- "Cold from knees to feet".

- "Most of the body is either dark blue or dark red. Cyanosis is especially severe on lips, fingernails and toenails".

observation to further characterise the patient's perspective of living with $\mathrm{PH}$.

\section{Analysis}

From the observational footage collected, a $45 \mathrm{~min}$ ethnographic film was produced for each country (seven in total) following analysis sessions between the researchers and analysts and healthcare experts within the research agency. A single 1-h cross-country film highlighting the key findings across the countries was also produced, and edited thematically to allow for crosscultural comparison. Footage was transcribed into text in English, so that the core team was able to read and compare all the interviews, and supplemented by field notes and patient diaries. Key themes from the research were derived through coding of footage and transcripts, which was undertaken by different members of the project team to ensure comprehensive identification of all key themes. Themes and reported behaviour were categorised by daily routines, impact on lifestyle, future outlook, information and support sources, medication practicalities, compliance and emotional attachment to therapy. Through a comparison of behavioural themes and articulated responses, the impact of $\mathrm{PH}$ on the patient's life was analysed to compare differences between reported behaviour and actual behaviour. Subgroup analysis was not performed.

\section{RESULTS}

\section{Patient demographics and data collection}

A total of 39 patients from seven countries were enrolled in the study, of whom 34 had been diagnosed with PAH and 5 with CTEPH (table 2). Patients were aged 19-91 years; the majority had New York Heart Association (NYHA) functional class II or III symptoms and approximately $75 \%$ were female. All enrolled patients completed the study.

Overall, $140 \mathrm{~h}$ of observational footage were collected and transcribed into 130 pages of text. This was supplemented with 36 pages of field notes and 28 patient diaries. The results are described under the following seven recurrent topics.

\section{The patient journey}

The patients' perspectives of their QoL changed dramatically between the 'prediagnosis', 'diagnosis' and 'living with PH' phases of their disease (figure 1). Many patients remained undiagnosed for several years, which they remembered as an emotional benchmark throughout their journey. Such patients experienced escalating symptoms, leading to a perceived steady decline in their QoL up until the point of diagnosis. Following diagnosis, patients were suddenly placed into a position of 'enforced dependency', which changed their relationship dynamics as partners, children and family members became caregivers. Access to medication played an integral role in their lives, providing symptomatic relief and vastly improving their QoL. Thereafter, QoL remained relatively stable, although monitoring disease progression was sometimes difficult as patients were unable to

Table 2 Patient demographics

\begin{tabular}{|c|c|c|c|c|c|c|c|c|c|}
\hline \multirow[b]{2}{*}{ Country } & \multicolumn{2}{|c|}{ PH type } & \multicolumn{4}{|c|}{ NYHA FC } & \multicolumn{2}{|c|}{ Gender } & \multirow[b]{2}{*}{ Age range, years } \\
\hline & $\overline{\text { PAH }}$ & CTEPH & I & II & III & IV & Male & Female & \\
\hline Brazil & 5 & 0 & 1 & 1 & 2 & 1 & 1 & 4 & $29-65$ \\
\hline France & 5 & 0 & 0 & 2 & 3 & 0 & 2 & 3 & $53-67$ \\
\hline Germany & 4 & 1 & 0 & 2 & 2 & 1 & 1 & 4 & $40-59$ \\
\hline Italy & 4 & 0 & 0 & 3 & 1 & 0 & 2 & 2 & $28-54$ \\
\hline Republic of Korea & 4 & 1 & 0 & 1 & 3 & 1 & 0 & 5 & $23-42$ \\
\hline UK & 3 & 2 & 0 & 2 & 2 & 1 & 2 & 3 & $19-75$ \\
\hline USA & 9 & 1 & 1 & 3 & 4 & 2 & 2 & 8 & $47-91$ \\
\hline Total & 34 & 5 & 2 & 14 & 17 & 6 & 10 & 29 & $19-91$ \\
\hline
\end{tabular}

CTEPH, chronic thromboembolic pulmonary hypertension; NYHA FC, New York Heart Association functional class; PAH, pulmonary arterial hypertension; $\mathrm{PH}$, pulmonary hypertension. 
Figure 1 The patient journey prediagnosis and postdiagnosis (HCP, healthcare professional; $\mathrm{PH}$, pulmonary hypertension).
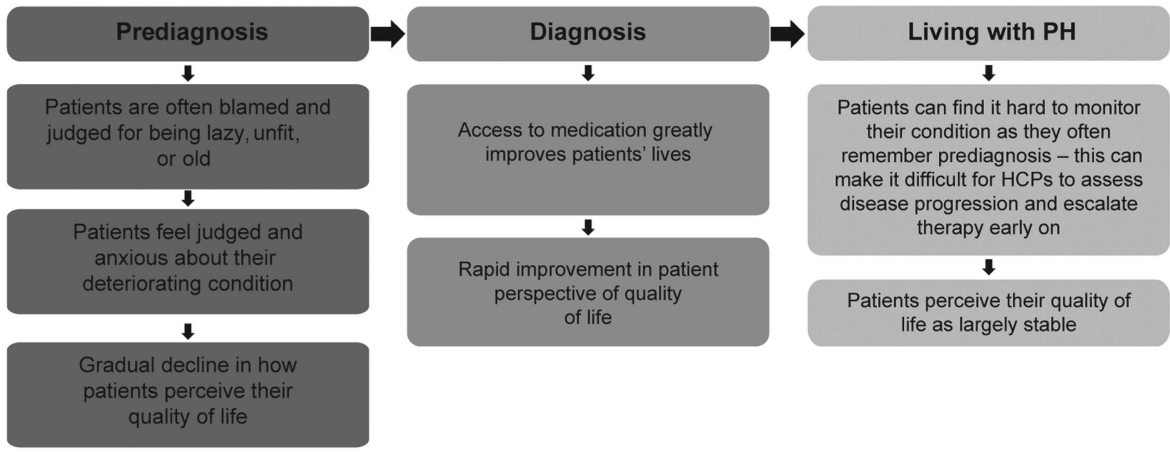

appreciate subtle differences in their symptoms. One patient stated: "Sometimes for fun, it slackens its grip and I think that it has gone forever, only for it to cruelly re-assert its control over me and I gasp for breath once more".

\section{The secretive nature of $\mathrm{PH}$}

Patients did not fully understand the meaning of their disease and there was no concise definition of $\mathrm{PH}$ that was easily understood. For example, one patient described being "Forced to carry a burden no one wants, or understands fully". Consequently, patients were uncomfortable talking about their disease, which in some cases prevented them from discussing it with family and friends. Words such as 'alone', 'sad' and 'stranger' were used by patients to describe $\mathrm{PH}$ if it was a person. The invisible nature of $\mathrm{PH}$ caused concern about how others perceived them and also made it difficult for patients to come to terms with their disease. Many patients admitted to hiding their symptoms, with this secrecy leading to feelings of isolation and depression. For instance, the following statement was recorded in one patient's diary: "PH has isolated me from the world. I want to rip this shackle off". Patients who had a connection with their national $\mathrm{PH}$ association reported feeling less isolated.

\section{Self-consciousness of PH}

Patients commonly described their embarrassment when having to stop to catch their breath in public as it attracted unwanted attention. As a coping strategy, many patients developed distraction routines, such as window shopping or looking at architecture. Some patients described their experiences of being labelled as 'lazy', 'unfit' or 'old', while other patients reported a perception of being judged as such. The visible nature of some medications, such as oxygen cylinders, inhalation devices and infusion pumps, also made patients feel selfconscious in public.

\section{Personality and daily routine}

The symptoms of PH limited a patient's lifestyle, and their personality often changed accordingly, as illustrated by the following comment from a patient: "If my illness was a person it would probably feel quite depressed and angry at times, because it could not do what it wants to do when it wants to do it". Many patients also reported deliberately adapting their personality to match the limitations placed on them by their disease, using phrases such as "I don't like sports", "going out isn't for me" and "I like staying in".

Older patients attempted to rationalise their symptoms by attributing them to the ageing process. In contrast, younger patients participated in less strenuous activities, and many developed hobbies, such as photography and writing, to match their activity tolerance. The research also highlighted the everyday difficulties of living with $\mathrm{PH}$ such as the fear of climbing stairs because of the effect it had on the body. Some patients were observed climbing stairs quickly, refusing to let their disease 'dominate' them, while others accepted their disease and climbed the stairs at a leisurely pace. When asked what would be their perfect day, it was clear that patients missed physical activities such as walking, running and swimming, as well as going outside with family and friends. Example responses included: "to hold out my hands and embrace everything I need to enjoy my day", "be able to get out and see lovely scenes" and "walk up a hill, climb a mountain, and not feel breathless".

In terms of disease-coping strategies, patients fell into two categories (figure 2). Solution seekers developed tactics to cope with $\mathrm{PH}$ on a daily basis, were less dependent on caregivers and were generally optimistic and motivated. This resulted in these patients maintaining a social life and being able to work part time. Conversely, disease-dominated patients had a more

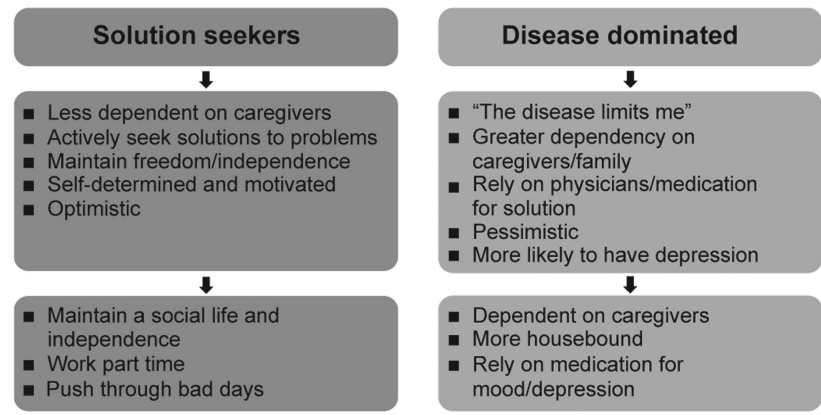

Figure 2 Patient types identified in relation to coping strategies. 
passive attitude towards $\mathrm{PH}$, a greater dependency on caregivers, a reliance on medication and were generally more pessimistic and more likely to experience depression. For example, one disease-dominated patient stated, "the disease limits me". Regardless of the coping strategy adopted, patients stated that extensive planning and adherence to daily routines were essential in everyday life and made statements such as "Walking is difficult so when I have to meet someone I have to leave the house early" and "My feet are stuck in mud but with patience I can get them out".

\section{Interaction with HCPs}

Patients regularly emphasised the importance of $\mathrm{PH}$ experts when describing their interactions with HCPs. One patient stated: "At the beginning, well, I of course still had such problems and then we spoke to Dr X on the phone most days. He was really afraid for me". Most patients had established a relationship with one key HCP who was usually an 'expert' in PH working in a specialist clinic or hospital (box 2). They had no preference for whether this 'expert' was a nurse or physician, so long as they had expert knowledge and were easily accessible. "It was back in 2003. I've been writing him an SMS message on every 7 th October for 8 years now. I write 'thank you...you've made me live again'."

Many patients were unable to recall details of consultations and appeared to remember what they wanted to hear. In the absence of a good relationship with HCPs, $\mathrm{PH}$ associations were an additional valuable source of advice and guidance. Most patients were aware of $\mathrm{PH}$ associations but not all patients utilised them. Caregivers were also considered an important source for emotional and practical support, as well as helping with medication administration.

\section{Relationship with medication}

Access to medication was crucial to patients' lives, and, as a result, a strong emotional attachment was evident. For example, one patient stated, "This is the most important drug, ever". Compliance to treatment was

Box 2 Example of a patient's response to the written task in their patient diary titled 'letter to my doctor'.

"Dear Doctor,

I have always felt that I could ask and discuss my condition with you and the people who look after me so well at my 3 monthly appointments at the...hospital. Perhaps I would if I felt the need, ie, that my condition becomes worse, ask you what the long-term and short-term effects would be.

Would I lose my quality of life and become more dependent on my husband and family or with medication can I hope and expect to carry on as I am? I know I would like to think that, as I have had this condition for the last 18 years. Can I be optimistic and hope that I can sustain this level and still look forward to a future of independence? At this moment in time, I am still smiling!" high and was structured into patients' daily routines. Non-compliance was often related to side effects, which patients recognised as different from the symptoms of PH: "If I was to sum it up, I'd say my PH is worse when I leave the house, but when I'm at home the side effects are worse". Patients tended to remember the "undiagnosed years' as a benchmark, which reinforced how important medication was to them. Thus, the marked improvement in symptoms after initiation of therapy made further assessment of subtle disease progression more difficult. Patients rarely had knowledge of a 'treatment plan', or how their treatment would change over time. When patients were asked how their treatment made them feel at present, responses included: "I can do everything I want and feel good!" and "to enjoy life and look at the bright side". In terms of how they hoped their treatment would make them feel in 6 months' time, patients highlighted their desire to "have a good quality of life" and "be able to go on holiday".

\section{Prognosis}

Patients reported receiving very different diagnoses and prognoses from different HCPs. They were often given a very negative outlook about their disease progression, but remained stable for longer than expected. This resulted in patients feeling 'uncertain' and 'hopeless', and some described their PH as being "always there". Many had not discussed their future with an HCP and claimed to be unaware of how their disease was likely to progress. Few expected their medication to cure them.

\section{DISCUSSION}

\section{Summary of main findings}

Despite the widespread use of QoL assessments in PH research, large gaps remain in our awareness of how patients perceive their disease. The present qualitative study used ethnography to gain a unique, real-life insight into $\mathrm{PH}$ from the patient's perspective. It uncovered a number of important findings, including the selfconsciousness and secretive nature of $\mathrm{PH}$, the difficulties that patients have in describing their disease, the emotional attachment to medication by patients and the challenges associated with assessing disease progression. Furthermore, it revealed the psychological aspects of the disease and the extensive planning and daily routines that are required to cope with everyday life.

\section{Meaning and implications}

One of the most striking themes that emerged from this study was the secrecy surrounding $\mathrm{PH}$, which was in part due to patients not fully understanding the meaning of $\mathrm{PH}$ and having difficulty in explaining it to others. A more tailored dissemination of information from HCPs and development of a simple and understandable definition of PH may therefore prove beneficial to patients and the public. The psychological impact of $\mathrm{PH}$ arises from the delay in diagnosis, the uncertainty of the 
disease, the progression of symptoms, the distress of breathlessness and, in many cases, the realisation that there is no cure. ${ }^{7}$ Feelings of isolation are common ${ }^{10-12}$; more than half of the patients with $\mathrm{PAH}$ and one-third of the carers in a European survey reported feeling isolated, mainly due to a lack of understanding of the disease among family, friends and the general public. ${ }^{13}$ In the present study, such feelings were particularly prominent in patients who admitted to hiding their symptoms. Fear and uncertainty about the disease process and self-consciousness, in terms of symptoms and visible forms of medication, were also reported here and in previous studies. ${ }^{1114}$ Thus, a greater appreciation of the psychological impact of PH by HCPs and caregivers could have a major influence on the patient's perceived QoL. It is therefore vital that HCPs educate patients about the disease process, their symptoms and the effect on QoL, encouraging participation in support groups, $\mathrm{PH}$ associations and counselling if appropriate. ${ }^{15}$

The rigorous planning and strict adherence to daily routines reported by patients in the present study is a common theme expressed by patients with $\mathrm{PH} .{ }^{15}{ }^{16}$ To this end, the most interesting outcome of this study was the extent to which patients adapted their lives to $\mathrm{PH}$, altering their personality and shaping new hobbies around their activity limitations. US and European surveys have also demonstrated the profound detrimental impact of PAH on QoL, including social life, physical and emotional well-being, sexual relationships and ability to work. ${ }^{13} 17$ Moreover, the US survey showed that only three-quarters were satisfied with their care from a QoL point of view. ${ }^{17}$ Thus, there may be a place in $\mathrm{PH}$ management for patient- and family-centred care that optimises QoL by addressing physical, intellectual, emotional, social and spiritual needs. ${ }^{17}$

Patients in this study emphasised the importance of interacting with expert $\mathrm{PH}$ physicians or nurses. A lack of faith in non-specialised HCPs may stem from the long periods of time that some patients spend undiagnosed and the misdiagnoses that they may have received. ${ }^{11} 18$ Referral to a specialist PH centre may ease patients' uncertainty as they will finally receive answers to longstanding questions. ${ }^{18}$ European treatment guidelines recommend that patients should always be referred to expert centres when $\mathrm{PH}$ is suspected, where support from a multidisciplinary team is available. ${ }^{1}$ The role of PH associations should also not be underestimated. ${ }^{1}$ In previous studies, patients have expressed almost universal appreciation for the role of $\mathrm{PH}$ associations in providing support, allowing them to connect with their fellow patients and gain a greater understanding of how $\mathrm{PH}$ affects their body. ${ }^{11}$

Numerous clinical and qualitative studies have previously shown that PAH-specific medications improve QoL in patients with $\mathrm{PAH}^{16}$ 19-22; however, the significant emotional attachment to medication reported in the present study is a novel finding. Although problems with tolerability and invasive administration methods have previously been reported to negatively impact $\mathrm{QoL},{ }^{15}$ tolerability and compliance were not reported to be major issues in this study. Nevertheless, the difficulty in assessing disease progression reported by patients and their lack of knowledge regarding long-term treatment plans has implications for long-term management. Such patients might be less likely to take a proactive approach to their illness, challenge treatment plans and discuss therapy escalation with their doctor. These difficulties may also delay the initiation of interventions to circumvent disease progression by physicians, which is key to successful disease management. ${ }^{1}{ }^{23}$

\section{Strengths and limitations}

To the best of our knowledge, this is the first ethnographic study to investigate the experiences of patients living with $\mathrm{PH}$. In order to provide an international sample and a wide range of perspectives on living with the disease, purposive sampling was utilised, including patients with PAH or CTEPH of varying severities and with a wide range of demographic features. This sample aimed to improve the understanding of the lives of patients with $\mathrm{PH}$, but it was not intended to be statistically representative of the $\mathrm{PH}$ population. In a healthcare setting, ethnography offers several advantages over other more commonly used qualitative techniques such as interviews and focus groups. By observing actual behaviour in the patient's environment, for relatively long periods of time, the researcher gains unique insight into the patient's perspective of their disease, observing the parts of life that are rarely considered by other qualitative techniques. Ethnography allows meaning to be created out of the mundane routines of everyday life, which may lead to the identification of solutions to improve QoL.

Nevertheless, the study has a number of limitations that could be addressed in future studies. Certain aspects of the study relied on the patients' retrospective analysis of their QoL during the prediagnosis and diagnosis phases of their illness. These accounts may benefit from hindsight and may or may not accurately reflect what actually happened. The sample size $(n=39)$ was too small to allow any subanalyses to be undertaken according to demographic variables, country or disease type, and this was not a major aim of this study. Investigating the experiences of older versus younger patients may be of interest in future studies, as European registry data indicate that elderly patients with $\mathrm{PH}$ (aged $\geq 65$ years) are less likely to reach treatment targets. $^{24}$ Additionally, the study could be extended to include caregivers and/or HCPs to assess how their perspectives compare with those of the patients. Although the current study was multinational, larger samples within a quantitative study would allow assessment of the statistical validity of the current findings.

\section{CONCLUSION}

In conclusion, this study provides a unique real-life insight into $\mathrm{PH}$ from the patient's perspective, 
uncovering a number of findings that would not typically be revealed by other qualitative approaches. A greater appreciation by HCPs and caregivers of how patients perceive their disease and their QoL has the potential to improve management of this chronic and debilitating disease. Ethnography appears to be a useful technique for assessing $\mathrm{PH}$ from the perspective of the patient and may be considered for future studies.

Contributors $\mathrm{BH}$ and OS were involved in study conceptualisation and design. $\mathrm{MK}, \mathrm{BH}, \mathrm{OS}$ and J-LV analysed and interpreted the results; and MK, BH, OS and J-LV prepared the manuscript.

Funding The study was carried out by Ipsos MORI (London, UK) and supported by Bayer HealthCare Pharmaceuticals (Berlin, Germany). Editorial assistance was provided by Adelphi Communications Ltd (Bollington, UK), supported by Bayer HealthCare Pharmaceuticals.

Competing interests MK has served on advisory boards and/or steering committees for Actelion Pharmaceuticals, Gilead Sciences, United Therapeutics Corporation and Bayer HealthCare Pharmaceuticals; she has also served as a speaker for Actelion Pharmaceuticals, Gilead Sciences and United Therapeutics Corporation. BH is a full-time employee of Bayer HealthCare Pharmaceuticals. OS is a full-time employee of Ipsos MORI. J-LV has served on advisory boards and/or steering committees for Actelion Pharmaceuticals, United Therapeutics Corporation and GlaxoSmithKline; he has received research support from GlaxoSmithKline and has also served as a speaker for Actelion Pharmaceuticals, Bayer HealthCare Pharmaceuticals,

GlaxoSmithKline, Eli Lilly and United Therapeutics Corporation.

Patient consent Obtained.

Ethics approval Local recruitment laws were adhered to.

Provenance and peer review Not commissioned; externally peer reviewed.

Data sharing statement We are able to make research materials available to view by a third party, which include the recruitment screeners, discussion guides and patient diaries. These extra materials are available by emailing $\mathrm{BH}$ (barbara.hinzmann@bayer.com). We are not able to share the transcripts of the research interviews as these were destroyed 6 months after the project end date, in accordance with Ipsos MORI's accreditation to the IS0:9001:2008 standard for Quality Management Systems. Furthermore, the research participants consented that their ethnographic footage would not enter the public domain and would only be used in communicating the research findings to Bayer HealthCare Pharmaceuticals by Ipsos MORI. There may be occasions whereby a third party could audit the footage to assess findings, but this would have to be arranged under clearly defined guidelines.

Open Access This is an Open Access article distributed in accordance with the Creative Commons Attribution Non Commercial (CC BY-NC 3.0) license, which permits others to distribute, remix, adapt, build upon this work noncommercially, and license their derivative works on different terms, provided the original work is properly cited and the use is non-commercial. See: http:// creativecommons.org/licenses/by-nc/3.0/.

\section{REFERENCES}

1. Galiè N, Hoeper MM, Humbert M, et al. Guidelines for the diagnosis and treatment of pulmonary hypertension: the task force for the diagnosis and treatment of pulmonary hypertension of the European
Society of Cardiology (ESC) and the European Respiratory Society (ERS), endorsed by the International Society of Heart and Lung Transplantation (ISHLT). Eur Heart J 2009;30:2493-537.

2. Benza RL, Miller DP, Barst RJ, et al. An evaluation of long-term survival from time of diagnosis in pulmonary arterial hypertension from REVEAL. Chest 2012;142:448-56.

3. Condliffe R, Kiely DG, Gibbs JS, et al. Improved outcomes in medically and surgically treated chronic thromboembolic pulmonary hypertension. Am J Respir Crit Care Med 2008:177:1122-7.

4. Mayer E, Jenkins D, Lindner J, et al. Surgical management and outcome of patients with chronic thromboembolic pulmonary hypertension: results from an international prospective registry. J Thorac Cardiovasc Surg 2011;141:702-10.

5. Pepke-Zaba J, Delcroix M, Lang I, et al. Chronic thromboembolic pulmonary hypertension (CTEPH): results from an International Prospective Registry. Circulation 2011;124:1973-81.

6. Gihl AF. Health-related quality of life in pulmonary arterial hypertension. Adv Pulm Hypertens 2010;8:215-22.

7. Rubenfire M, Lippo G, Bodini BD, et al. Evaluating health-related quality of life, work ability, and disability in pulmonary arterial hypertension: an unmet need. Chest 2009;136:597-603.

8. NHS Oldham UK. Oldham Public Health Report. 2008.

9. Savage J. Ethnography and health care. BMJ 2000;321:1400-2.

10. Lowe B, Grafe K, Ufer C, et al. Anxiety and depression in patients with pulmonary hypertension. Psychosom Med 2004;66:831-6.

11. Studer SM, Migliore C. Quality of life in pulmonary arterial hypertension: qualitative insights from patients and caregivers. $A d v$ Pulm Hypertens 2012;10:222-6.

12. Wryobeck JM, Lippo G, McLaughlin V, et al. Psychosocial aspects of pulmonary hypertension: a review. Psychosomatics 2007;48: 467-75.

13. Aldrighetti R, Howard L, Armstrong I, et al. The impact of pulmonary arterial hypertension (PAH) on the lives of patients and carers: results from an international survey. http://www.phaeurope.org/ projects-activities/pah-patient-and-carer-survey/2012.

14. McDonagh TA, Cunningham AD, Morrison CE, et al. Left ventricular dysfunction, natriuretic peptides, and mortality in an urban population. Heart 2001;86:21-6.

15. McDonough A, Matura LA, Carroll DL. Symptom experience of pulmonary arterial hypertension patients. Clin Nurs Res 2011;20:120-34.

16. Flattery MP, Pinson JM, Savage L, et al. Living with pulmonary artery hypertension: patients' experiences. Heart Lung 2005;34:99-107.

17. Swetz KM, Shanafelt TD, Drozdowicz LB, et al. Symptom burden, quality of life, and attitudes toward palliative care in patients with pulmonary arterial hypertension: results from a cross-sectional patient survey. J Heart Lung Transplant 2012;31:1102-8.

18. Armstrong I, Rochnia N, Harries $\mathrm{C}$, et al. The trajectory to diagnosis with pulmonary arterial hypertension: a qualitative study. BMJ Open 2012;2:1-9.

19. Barst RJ, Rubin LJ, Long WA, et al. A comparison of continuous intravenous epoprostenol (prostacyclin) with conventional therapy for primary pulmonary hypertension. The Primary Pulmonary Hypertension Study Group. N Engl J Med 1996;334:296-302.

20. Keogh AM, McNeil KD, Wlodarczyk J, et al. Quality of life in pulmonary arterial hypertension: improvement and maintenance with bosentan. J Heart Lung Transplant 2007;26:181-7.

21. Olschewski H, Simonneau G, Galiè N, et al. Inhaled iloprost for severe pulmonary hypertension. N Engl J Med 2002;347:322-9.

22. Wilkins MR, Paul GA, Strange JW, et al. Sildenafil versus endothelin receptor antagonist for pulmonary hypertension (SERAPH) study. Am J Respir Crit Care Med 2005;171:1292-7.

23. Vachièry $\mathrm{JL}$, Yerly $\mathrm{P}, \mathrm{Huez} \mathrm{S}$. How to detect disease progression in pulmonary arterial hypertension. Eur Respir Rev 2012;21:40-7.

24. Hoeper MM, Huscher D, Ghofrani HA, et al. Elderly patients diagnosed with idiopathic pulmonary arterial hypertension: results from the COMPERA registry. Int J Cardiol 2012;168:871-80. 